\title{
An Exactly Solvable Supersymmetric Model of Semimagic Nuclei
}

\author{
A. B. Balantekin*, Nurtaç Güven ${ }^{\dagger}$ and Yamaç Pehlivan ${ }^{\dagger}$ \\ * Department of Physics, University of Wisconsin, Madison, Wisconsin 53706 USA \\ ${ }^{\dagger}$ Department of Applied Mathematics, Haliç University, Istanbul 34104 Turkey
}

\begin{abstract}
A simple model of nucleons coupled to angular momentum zero (s-pairs) occupying the valance shell of a semi-magic nuclei is considered. The model has a separable, orbit dependent pairing interaction which dominates over the kinetic term. It is shown that such an interaction leads to an exactly solvable model whose $\left(0^{+}\right)$eigenstates and energies can be computed very easily with the help of the algebraic Bethe ansatz method. It is also shown that the model has a supersymmetry which connects the spectra of some semimagic nuclei. The results obtained from this model for the semimagic $\mathrm{Ni}$ isotopes from ${ }^{58} \mathrm{Ni}$ to ${ }^{68} \mathrm{Ni}$ are given. In addition, a new and easier technique for calculating the energy eigenvalues from the Bethe ansatz equations is also presented.
\end{abstract}

Keywords: Nuclear Pairing, Shell Model, Bethe Ansatz, Supersymmetry, Integrable Systems.

PACS: 21.60.Fw, 21.60.-n, 21.60.Cs, 02.30.Ik

We consider the $s$-pairs of the same kind of nucleons (either protons or neutrons) occupying the valance shell of a semi-magic nuclei and interacting with an orbit dependent separable pairing force as described by the Hamiltonian

$$
H=\sum_{j} \sum_{m} \varepsilon_{j} a_{j m}^{\dagger} a_{j m}-|G| \sum_{j j^{\prime}} c_{j}^{*} c_{j^{\prime}} S_{j}^{+} S_{j^{\prime}}^{-} .
$$

The orbit dependence of the pairing force is described by the dimensionless constants $c_{j}$ while the overall strength of the pairing term against the kinetic term is measured by the constant $|G|$ which has the dimension of energy. In writing the Hamiltonian (1), we use the quasispin operators which are given by [1]

$$
S_{j}^{+}=\left(S_{j}^{-}\right)^{\dagger}=\sum_{m>0}(-1)^{j-m} a_{j m}^{\dagger} a_{j-m}^{\dagger} \quad S_{j}^{0}=\frac{1}{2} \sum_{m>0}\left(a_{j m}^{\dagger} a_{j m}+a_{j-m}^{\dagger} a_{j-m}-1\right) .
$$

Quasispin operators obey the angular momentum commutation relations

$$
\left[S_{j}^{+}, S_{j^{\prime}}^{-}\right]=2 \delta_{j j^{\prime}} S_{j}^{0} \quad\left[S_{j}^{0}, S_{j^{\prime}}^{ \pm}\right]= \pm \delta_{j j^{\prime}} S_{j}^{ \pm},
$$

i.e., one has an angular momentum algebra for each orbit $j$ such that those angular momenta corresponding to different orbits commute with one another. Note that Eq. (2) implies $-\Omega_{j} / 2 \leq S_{j}^{0} \leq \Omega_{j} / 2$ where $\Omega_{j}=j+1 / 2$ is the maximum number of $s$-pairs which can occupy the orbit $j$. This tells us that the quasi-spin algebra corresponding to the level $j$ is realized in the $\Omega_{j} / 2$ representation.

Here, we are interested in the limit of the pairing Hamiltonian in which all the single particle energy levels become degenerate. In this degenerate limit, the pairing 
Hamiltonian has two interesting properties which are discussed in this talk: 1) It is exactly solvable and 2) it is invariant under a supersymmetry transformation which connects the spectra of different isotopes or isotones. Note that the pairing Hamiltonian is also exactly solvable away from the degenerate limit (i.e., with different single particle energy levels) but only in the presence of two orbits [2]. From this solution, we know that the supersymmetry mentioned above is broken away from the degenerate limit.

We begin by noting that in the degenerate limit, the one body term of the pairing Hamiltonian is reduced a constant proportional to the total number of pairs which can be discarded. Therefore, in the degenerate limit, the pairing Hamiltonian becomes

$$
\hat{H}_{D}=-|G|\left(\sum_{j} c_{j}^{*} S_{j}^{+}\right)\left(\sum_{j^{\prime}} c_{j^{\prime}} S_{j^{\prime}}^{-}\right)
$$

This tells us that the degenerate limit of the pairing Hamiltonian can also be viewed as the strong pairing limit.

Exact eigenvalues and eigenstates of the Hamiltonian given in Eq. (4) were obtained in Refs. [3, 4, 5] (see Ref. [6] for the eigenvalues of the quantum invariants of this Hamiltonian). Following these references, we introduce the operators

$$
S^{+}(x)=\sum_{j} \frac{c_{j}^{*}}{1-\left|c_{j}\right|^{2} x} S_{j}^{+} \quad \text { and } \quad S^{-}(x)=\sum_{j} \frac{c_{j}}{1-\left|c_{j}\right|^{2} x} S_{j}^{-} .
$$

These operators respectively create and annihilate a single pair of nucleons in the valance shell in a certain distribution over the single particle energy levels described by the complex parameter $x$. The eigenstates of the pairing Hamiltonian in the degenerate limit can be written in terms of these operators. For example, in Ref. [7] Talmi has showed that, in the presence of one pair of nucleons in the valance shell,

$$
\hat{S}^{+}(0)|0\rangle \quad E=-|G| \sum_{j} \Omega_{j}\left|c_{j}\right|^{2}
$$

is an eigenstate-eigenvalue pair of the Hamiltonian (4). Additional eigenstates with one pair of nucleons can be found by considering a more general Bethe ansatz state in the form of $\hat{S}^{+}(x)|0\rangle$. In fact, using the quasi-spin angular momentum algebra given in Eq. (3), it is easy to show that

$$
\hat{S}^{+}(x)|0\rangle \quad E=0
$$

is an eigenstate-eigenvalue pair of the Hamiltonian (4) if the parameter $x$ is a solution of the Bethe ansatz equation

$$
\sum_{j} \frac{-\Omega_{j} / 2}{1 /\left|c_{j}\right|^{2}-x}=0
$$

Note that Eq. (8) may have several distinct solutions in which case we have a zero energy eigenstate in the form of Eq. (7) for each solution. It is worth to emphasize at this point that all the energies are calculated with respect to the core and that we have ignored the one body term in the Hamiltonian. 
These considerations generalize to the case of several pairs occupying the valance shell. Let us denote the maximum number of pairs which can occupy the valance shell by $N_{\max }$. If the shell is occupied by $N$ pairs such that $N \leq N_{\max } / 2$ then the pairing Hamiltonian given in Eq. (4) has some eigenstates with nonzero energy and some eigenstates with zero energy, similar to the one pair case discussed above [3, 4, 5]. The nonzero energy eigenstates together with their energies are given by

$$
\hat{S}^{+}(0) \hat{S}^{+}\left(z_{1}\right) \ldots \hat{S}^{+}\left(z_{N-1}\right)|0\rangle \quad E=-|G|\left(\sum_{j} \Omega_{j}\left|c_{j}\right|^{2}-\sum_{k=1}^{N-1} \frac{2}{z_{k}}\right)
$$

where the parameters $z_{1}, z_{2}, \ldots, z_{N-1}$ are solutions of the set of Bethe ansatz equations

$$
\sum_{j} \frac{-\Omega_{j} / 2}{1 /\left|c_{j}\right|^{2}-z_{m}}=\frac{1}{z_{m}}+\sum_{k=1(k \neq m)}^{N-1} \frac{1}{z_{m}-z_{k}}
$$

for $m=1,2, \ldots, N-1$. Similarly, the zero energy eigenstates are given by

$$
\hat{S}^{+}\left(x_{1}\right) \hat{S}^{+}\left(x_{2}\right) \ldots \hat{S}^{+}\left(x_{N}\right)|0\rangle \quad E=0
$$

where the parameters $x_{1}, x_{2}, \ldots, x_{N}$ are solutions of the Bethe ansatz equations

$$
\sum_{j} \frac{-\Omega_{j} / 2}{1 /\left|c_{j}\right|^{2}-x_{m}}=\sum_{k=1(k \neq m)}^{N} \frac{1}{x_{m}-x_{k}}
$$

for $m=1,2, \ldots, N$. Note that the Bethe ansatz equations given above for both the nonzero energy eigenstates and the zero energy eigenstates may have several distinct sets of solutions. In such a case, each solution set gives us an eigenstate-eigenvalue pair when substituted in the relevant formulas. In particular, if the zero energy Bethe ansatz equations have more than one solutions then the zero energy level is degenerate.

Let us now consider a valance shell which is more than half full, i.e., $N>N_{\max } / 2$. One can, in principle, extend the formulas (9-12) for $N>N_{\max } / 2$. But in this case one frequently encounters one of the following two problems: 1) The Bethe ansatz equations given above yield no solutions or 2) the Bethe ansatz equations do yield solutions but the corresponding states vanish identically. For this reason, it is more reasonable to use hole pairs instead of nucleon pairs to describe a valance shell which is more than half full.

The pairing Hamiltonian given in Eq. (4) is invariant under a supersymmetry transformation which transforms the spectrum of a nuclei with $N$ pairs of valance nucleons $\left(N \leq N_{\max } / 2\right)$ to the spectrum of a nuclei with $N-1$ pairs of valance holes. In order to describe this symmetry, let us first introduce the particle-hole transformation operator

$$
\hat{T}=\exp \left(-i \pi \sum_{j} \frac{\hat{S}_{j}^{+}+\hat{S}_{j}^{-}}{2}\right)
$$

$\hat{T}^{\dagger}$ transforms the empty valance shell $|0\rangle$ into the fully occupied valance shell $|\overline{0}\rangle$ and the pair creation-annihilation operators into each other, i.e.,

$$
\hat{T}^{\dagger}|0\rangle=|\overline{0}\rangle \quad \hat{T}^{\dagger} S_{j}^{ \pm} \hat{T}=S_{j}^{\mp} \quad \hat{T}^{\dagger} S_{j}^{0} \hat{T}=-S_{j}^{0}
$$


Therefore, it transforms a state containing $N$ particle-pairs into states containing $N$ holepairs. However, there is no particle-hole symmetry in the problem described by the pairing Hamiltonian as can be easily verified by showing that $\left[H_{D}, \hat{T}\right] \neq 0$. On the other hand, if we define the operators

$$
\hat{B}^{+}=\left(\sum_{j} c_{j}^{*} \hat{S}_{j}^{+}\right) \hat{T} \quad \hat{B}^{-}=\hat{T}^{\dagger}\left(\sum_{j} c_{j} \hat{S}_{j}^{-}\right)
$$

then it is easy to show that $\hat{B}^{+}$and $\hat{B}^{-}$commute with one another

$$
\left[\hat{B}^{+}, \hat{B}^{-}\right]=0
$$

and that the pairing Hamiltonian given in Eq. (4) can be written as

$$
\hat{H}_{D}=-|G| \hat{B}^{-} \hat{B}^{+}=-|G| \hat{B}^{+} \hat{B}^{-} .
$$

This tells us that the problem described by the pairing Hamiltonian in the degenerate limit possesses a quantum mechanical supersymmetry. Since the operators $\hat{B}^{+}$and $\hat{B}^{-}$commute with each other, the Hamiltonian $\hat{H}_{D}$ is equal to its own supersymmetric partner. As a result, if the state $|\psi\rangle$ is an eigenstate of the Hamiltonian $\hat{H}_{D}$ with a nonzero energy, then the state $\hat{B}^{-}|\psi\rangle$ is also an eigenstate of $\hat{H}_{D}$ with the same energy. Zero energy states, however, are annihilated by $\hat{B}^{-}$and do not transform under the supersymmetry. It is easy to see from the definition of $\hat{B}^{-}$given in Eq. (15)

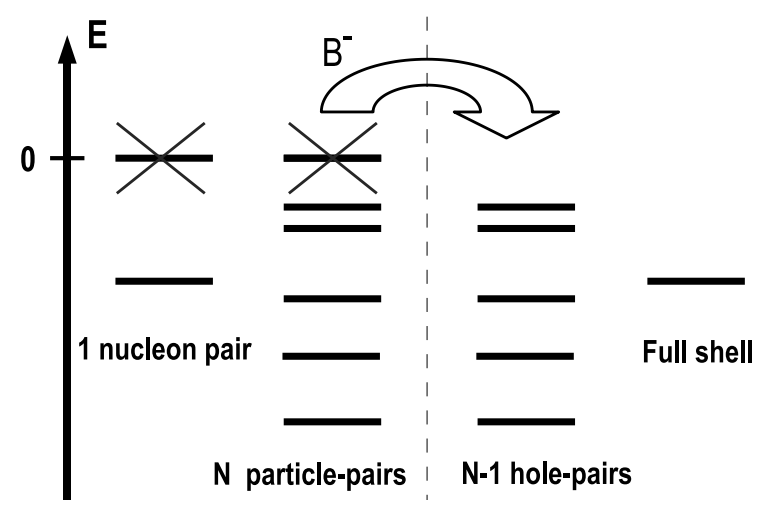
that if the state $|\psi\rangle$ has $N$ particle-pairs, then the state $\hat{B}^{-}|\psi\rangle$ has $N-1$ hole-pairs. Because the operator $\hat{B}^{-}$first annihilates one particle-pair and then turns the remaining $N-1$ particle-pairs into hole-pairs. Consider, for example, the nonzero energy eigenstate with one pair of particles given in Eq. (6). Using Eqs. (14) and (17), one can easily show that

$$
\hat{B}^{-} \hat{S}^{+}(0)|0\rangle \propto|\overline{0}\rangle
$$

Consequently, the states $\hat{S}^{+}(0)|0\rangle$ and $|\overline{0}\rangle$ (i.e., the fully occupied valance shell) have the same energy eigenvalue given by Eq. (6). On the other hand, the zero energy eigenstates described by Eqs. (7) and (8) are annihilated by $\hat{B}^{-}$. Similarly, the nonzero energy eigenstates with $N$ pairs of nucleons described in Eqs. (9) and (10) transform as

$$
\hat{B}^{-} \hat{S}^{+}(0) \hat{S}^{+}\left(z_{1}\right) \ldots \hat{S}^{+}\left(z_{N-1}\right)|0\rangle \propto \hat{S}^{-}\left(z_{1}\right) \ldots \hat{S}^{-}\left(z_{N-1}\right)|0\rangle
$$

As a result, the states $\hat{S}^{+}(0) \hat{S}^{+}\left(z_{1}\right) \ldots \hat{S}^{+}\left(z_{N-1}\right)|0\rangle$ and $\hat{S}^{-}\left(z_{1}\right) \ldots \hat{S}^{-}\left(z_{N-1}\right)|0\rangle$ have the same energy given in Eq. (9). Since $N \leq N_{\max } / 2$, the former state corresponds to a nucleus which has less than half full or half full valance shell 
whereas the later state corresponds to a nucleus which has more than half full valance shell (See Figure 1). Note that the zero energy states with $N$ pairs of nucleons described by Eqs. (11) and (12) are annihilated by $\hat{B}^{-}$. As an application of this formalism, $c_{1 / 2}=\frac{0.315 \quad}{2 \mathrm{p}_{1 / 2}} \Omega_{1 / 2}=1$ let us consider the even-even $\mathrm{Ni}$ isotopes from ${ }^{58} \mathrm{Ni}$ to ${ }^{68} \mathrm{Ni}$ for which the neutron and proton shells are closed at $c_{5 / 2}=\frac{0.545 \quad}{\Omega_{5 / 2}=\imath}$ $N=Z=28$ (i.e., the core is the doubly magic ${ }^{56} \mathrm{Ni}$ nucleus). The extra neutrons in ${ }^{58} \mathrm{Ni}^{68}{ }^{6} \mathrm{Ni}$ occupy the single par-

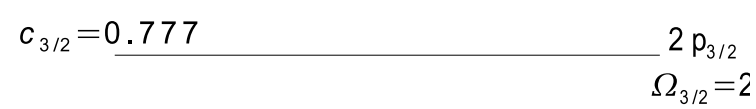

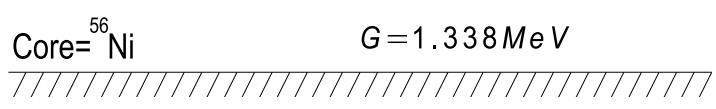
ticle energy levels shown in Figure 2. The occupation probability amplitudes shown in this figure are taken from Ref. [8] and the value of the pairing strength $|G|$ is chosen so as to obtain the ground state energy of ${ }^{58} \mathrm{Ni}$ (with respect to ${ }^{56} \mathrm{Ni}$

FIGURE 2. The valance shell for ${ }^{58} \mathrm{Ni}^{68} \mathrm{Ni}$. core) in agreement with the experimental data. Figure 3 shows the exact energy eigenvalues of the pairing Hamiltonian given in Eq. (4) calculated with the formalism outlined above. The zero energy states of ${ }^{58} \mathrm{Ni}$ and ${ }^{60} \mathrm{Ni}$ isotopes are doubly degenerate as indicated by double lines in the figure. All the energies are given in $\mathrm{MeV}$ and are relative to the ${ }^{56} \mathrm{Ni}$ core. We use the supersymmetry to obtain the spectra of the nuclei with more than half full shell (i.e., ${ }^{64} \mathrm{Ni}-{ }^{68} \mathrm{Ni}$ ). For example, the spectrum of ${ }^{64} \mathrm{Ni}$ is the same as the spectrum of ${ }^{60} \mathrm{Ni}$ except that the zero energy states are absent for ${ }^{64} \mathrm{Ni}$. See Ref. [4] for a comparison of these results with the experimentally available values.

With the Bethe ansatz formalism outlined above, one can easily calculate the exact numerical values of the pairing energies. However, to obtain closed analytical expressions for the pairing energies is relatively difficult in this formalism. Because this would require the triple effort of solving the Bethe ansatz equations analytically, substituting the solutions into the energy expression given in Eq. (9) and then making the necessary simplifications. In the last part, we present an easier method of calculating the energy eigenvalues. Although this method is currently valid for only two orbits, it can possibly be extended to include more than two orbits.

In the presence of only two orbits, the problem of calculating the nonzero energy eigenvalues can be greatly simplified by using a method proposed by Stieltjes [9]. Using this method, one can transform the problem of solving the equations (9) and finding the energy eigenvalues for $N$ nucleon-pairs into the problem of finding $N^{t h}$ order polynomial solutions of the following differential equation [10]:

$$
p_{N}^{\prime \prime}(z)-\left(\frac{\Omega_{j_{2}}}{z}+\frac{\Omega_{j_{1}}}{z-1}\right) p^{\prime}(z)+\frac{\left(\alpha \beta z-q_{N}\right)}{z(z-1)(z-c)} p_{N}(z)=0
$$

where $\alpha=-N, \beta=N-\Omega_{j_{1}}-\Omega_{j_{2}}-1$ and

$$
c=-\frac{1 / c_{j_{2}}^{2}}{1 / c_{j_{1}}^{2}-1 / c_{j_{2}}^{2}} \quad q=\frac{-E /|G|+N\left(N-\Omega_{j_{1}}-\Omega_{j_{2}}-1\right) c_{j_{1}}^{2}}{c_{j_{2}}^{2}-c_{j_{1}}^{2}} .
$$




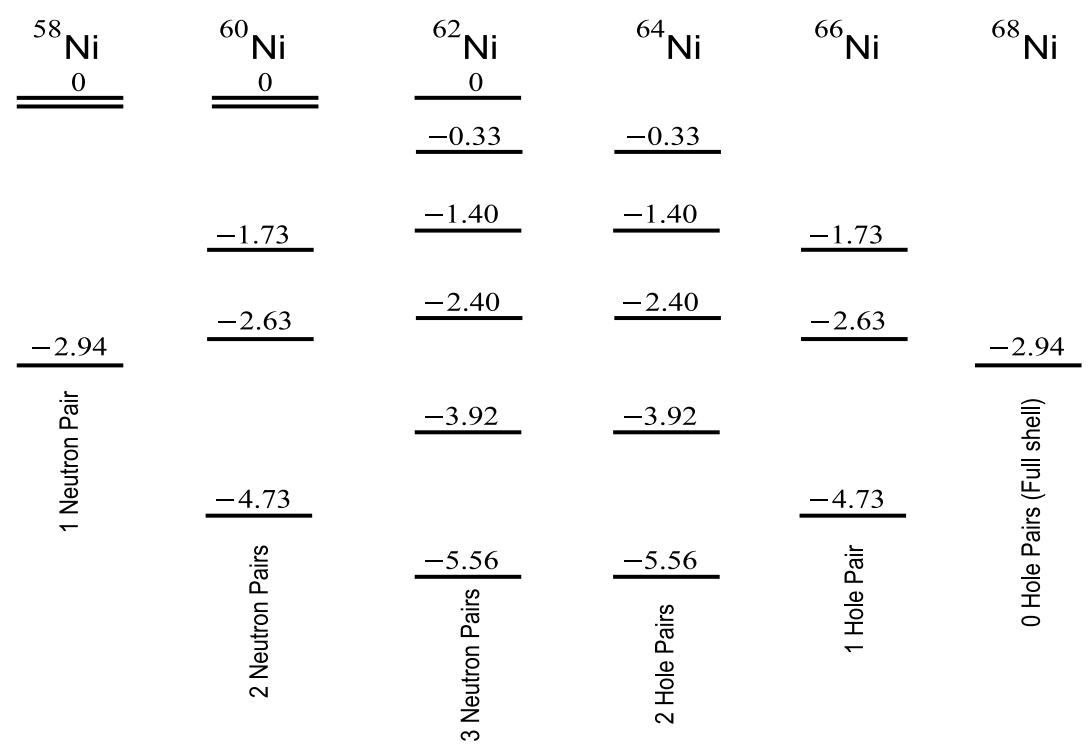

FIGURE 3. The exact energy eigenvalues of the pairing Hamiltonian for ${ }^{58} \mathrm{Ni}^{-6}{ }^{6} \mathrm{Ni}$ in $\mathrm{MeV}$ relative to the ${ }^{56} \mathrm{Ni}$ core. The degeneracies of the zero energy states of ${ }^{58} \mathrm{Ni}^{-60} \mathrm{Ni}$ are indicated by the double lines.

Note that a similar but slightly different method was used in Ref. [5] in order to obtain solutions of the zero energy Bethe ansatz equations. Eq. (20) is known as Heun's differential equation and it admits polynomial solutions only for certain values of the parameter $q$. One can use the Frobenius method (i.e., try a series solution) and then find the conditions on $q$ such that the series will terminate at the $N^{t h}$ order. This gives us the allowed values of the energy eigenvalues for $N$ pairs of nucleons because $q$ and $E$ are related by Eq. (21). This method significantly reduces the amount of calculation needed to compute the energy eigenvalues of the pairing Hamiltonian given in Eq. (4) [10].

This work was supported in part by the U.S. National Science Foundation Grant No. PHY-0555231 at the University of Wisconsin, and in part by the University of Wisconsin Research Committee with funds granted by the Wisconsin Alumni Research Foundation.

\section{REFERENCES}

1. A. K. Kerman, Ann. Phys. 12 (1961), 300.

2. A. B. Balantekin and Y. Pehlivan, Phys. Rev. C 76, 051001 (2007) [arXiv:0710.3941 [nucl-th]].

3. F. Pan, J. P. Draayer and W. E. Ormand, Phys. Lett. B 422, 1 (1998) [arXiv:nucl-th/9709036].

4. A. B. Balantekin, J. H. de Jesus and Y. Pehlivan, Phys. Rev. C 75, 064304 (2007) [arXiv:nucl-th/0702059].

5. A. B. Balantekin and Y. Pehlivan, J. Phys. G 34, 1783 (2007) [arXiv:0705.1318 [nucl-th]].

6. Y. Pehlivan, [arXiv:0806.1810 [math-ph]].

7. I. Talmi, Nucl. Phys. A172, 1 (1971)

8. N. Auerbach, Nuclear Physics 76 (1966) 321-335.

9. T.J. Stieltjes, 1914, Sur Quelques Theoremess d'Algebre, Oeuvres Completes, V. 11 (Groningen:Noordhoff).

10. N. Güven and Y. Pehlivan., in preparation.

11. A. Ronveaux, 1995, Heun's Differential Equations, (Oxford Science Publications). 